\title{
ANTECEDENTE NACIONAL E INTERNACIONAL SOBRE LA PERCEPCIÓN Y LOS DERECHOS DE LAS NIÑAS, LOS NIÑOS, LOS Y LAS ADOLESCENTES
}

\author{
Rodolfo Vicente Salazar \\ Académico del CIDE-INEINA, Universidad Nacional \\ Heredia, Costa Rica
}

Recibido 16 de octubre 2006 • Aprobado 14 de febrero 2007

\begin{abstract}
Resumen: Hasta mediados del siglo XVIII, el niño fue considerado como un adulto en miniatura; pero Juan Jacobo Rousseau abrió las puertas al estudio del ser en formación, y sentó las bases necesarias para la ciencia Paidología o estudio del niño, que enfoca los conflictos intelectuales y físicos de la vida infantil, y sus secuelas Paidofilaxis, Puericultura y Pediatría.

De la Sociedad de las Nacionales, surgió el Consultorio de la Trata de Mujeres y la Protección del Niño, en 1919, y en Ginebra se fundó la Unión Internacional de Socorros, en 1920.

En 1916, se realizó en Argentina el I Congreso Americano del Niño, al cual sucedieron otros regularmente en América. En 1924, se redactó la primera "Declaración de los Derechos del Niño" y luego se proclamó el primer decálogo referente a los Derechos del Niño.

En mayo de 1929, se presentó al Congreso Constitucional un proyecto de Ley para crear una institución que tuviera por objeto la protección del Niño, que se convirtió en Ley en 1930, con el nombre del Patronato Nacional de la Infancia (PANI), con el encargo de velar por el bienestar de las personas menores de edad en su desarrollo físico, intelectual, moral y emocional.

En 1930, en Costa Rica, se hizo la Primera Declaración de los Derechos del Niño Costarricense.

La creación del PANI, la Declaración de los Derechos del Niño, y la instauración del Código de la Infancia, son tres hechos que sitúan a Costa Rica como uno de los países pioneros en Latinoamérica y en el mundo, respecto a la protección que se les brinda al niño y a la madre.
\end{abstract}

Palabras clave: Protección de la niñez, Declaración de los derechos de la niñez.

\begin{abstract}
Until the middle of the XVIII Century, children were considered as miniature adults, but with Jean Jacob Rousseau the study of the development of the human being was started and the foundation was set up for the new science of Paidology (the study of children), wich focuses on the different intellectual and physical conflicts which children face, and also for other sciencies such as Paidofilaxis, Pueroculture and Pediatrics. The Society of Nations, created, in 1919, the Consultancy for the Trafficking of Women and the Protection of Children, and in Geneva, the International Union for Aid was founded in 1920. In Argentina, the First American Congress for Children was organized in 1916, and others followed. The first Declaration of Childrens Right was proclaimed in 1924 and then followed the first Ten Commandments of Childrens Rights.
\end{abstract}

\footnotetext{
Académico del CIDE-INEINA, Universidad Nacional, Heredia, Costa Rica. Especialista en Justicia, Políticas Públicas, Niñez y Adolescencia. . Consultor Save the Children, Suecia.
} 
In Costa Rica in 1929, a project was presented for the Constitutional Congress to establish an institution for the protection of children, wich was duly enacted into law in 1930 with the name of National Institution for the Protection of Children (Patronato Nacional de la Infancia, PANI) whose duty was to oversee the welfare of minors and their physical, intellectual, moral and emotional development. In 1930, the first Declaration of the Rights for Costarrican Children was proclaimed.

The creation of the PANI, the declaration of Childrens Rights, and the enactment of a Code for Infants (1932), constitute the three events that signal Costa Rica as one of the pioneers countries in Latin American and the World, in the protectionof children and mothers.

At the end of the article, some of the crucial events that have happened during the decades that PANI has been working in the country are analized.

Key words: Protection of chidren, Declaration of children Rights.

Apartir de mediados del siglo XVIII se dejó de pensar al niño como un adulto en miniatura. Juan Jacobo Rousseau abrió las puertas al estudio del ser humano en formación y una serie de médicos, pedagogos y psicólogos, sistematizaron sus observaciones, con los cual estructuraron las bases necesarias para una ciencia nueva: la Paidología o estudio del niño, que se enfoca en los conflictos intelectuales y físicos de la vida infantil.

Conforme se adelanta el estudio sobre ser niña y niño, desconocido por siglos, profesionales en diversos campos dirigieron su interés a penetrar íntimamente en su naturaleza, a seguir su desenvolvimiento; se conoció en el mundo entero una serie de problemas característicos de la niñez, cuya desatención redunda en una sociedad deficiente biológica, moral y psicológicamente.

Dada la naturaleza de la niña, el niño y el o la adolescente, en todos los ámbitos de nuestro planeta, tanto en circunstancias de civilización como de primitivismo, se le presenta a esta persona menor de edad, las mismas necesidades materiales y éticas. Por esta razón, los problemas de la infancia pueden catalogarse de manera general.

Dado que la preocupación por el desarrollo del ser humano en el siglo XX penetra en la conciencia de educadores, médicos, filósofos y legisladores, en el llamado siglo del NIÑO, toman auge una serie de actividades científicas y sociales tendientes a la protección y mejoramiento de la niñez.

Así, surge una ciencia nueva: la Paidofilaxis, que pretende, según lo menciona el Profesor don Luis Felipe González Flores (1931), en un documento del Patronato Nacional de la Infancia:

...la prevención de los males que afligen a la infancia; el estudio y la aplicación de los principios científicos destinados a asegurar en las mejores condiciones la gestación y el nacimiento del niño, así como su desarrollo integral, hasta el momento en que es capaz de bastarse así mismo y de ser un elemento activo y útil en la sociedad de que forma parte (p. 10).

En apoyo de una ciencia tan nueva, acuden otras de origen más antiguo, como la Biología, Anatomía, Eugenesia, Derecho, Sociología, Paidología y Puericultura. Como nota descollante, surge una rama específica de la Medicina: la Pediatría, encaminada a enmendar errores que en épocas pasadas costaron la vida de niñas, niños y adolescentes.

Es notorio el interés por el mejoramiento y la modernización de otra ciencia eminentemente consagrada a los intereses infantiles: la Pedagogía, cuyo fin es el de aplicar los métodos más apropiados para conseguir el pleno rendimiento de las potencialidades humanas. 
Las sociedades modernas, conscientes de la trascendencia social de los problemas que afectan a la infancia y del beneficio que para el futuro de las naciones significa su adecuada solución, se ha dado en casi todos los países, un movimiento redentor de la niñez, caracterizado por la búsqueda de una legislación que mejore y proteja la situación de las personas menores de edad, a quienes la vida haya colocado en situaciones desventajosas. En esta búsqueda de beneficios, se han organizado cónclaves internacionales propuestos a encontrar los medios para resolver el problema, puesto que sus proyecciones afectan de manera directa a toda la humanidad.

Así, al finalizar la Primera Guerra Mundial surgió, del seno de la Sociedad de las Naciones, en el Tratado de Versalles, en 1919, el Consultorio de la Trata de Mujeres y la Protección del Niño. En 1920, se fundó en Ginebra la Unión Internacional de Socorros, con miras a recoger fondos para la niñez abandonada y escasamente alimentada, sin distinción de raza, credo político, religión o nacionalidad.

En América, en 1920, se había reunido el Congreso Científico Internacional, del cual nació por idea del Doctor Antonio Vial, el Primer Congreso Americano del Niño, que se llevó a cabo en Buenos Aires, Argentina en 1916. En consecuencia, se organizó la Liga de los Derechos de la Mujer y del Niño, y ésta realizó el Primer Congreso del Niño en Argentina.

Al primer Congreso Americano del Niño, sucedieron: el II, celebrado en Montevideo en 1920; el III en Río de Janeiro, Brasil, en 1922; y el IV en Santiago de Chile, en 1924; el V en la Habana, Cuba, en 1927; el VI en Lima, Perú, en 1930; y el VII en México, en 1935. Como se ve, en América ha aumentado el interés por el bienestar de la infancia.

Si fuera oportuno un análisis de cuáles son los países del mundo que han marchado a la vanguardia, en su preocupación por las más eficaces soluciones a los problemas de la niñez, habría que mencionar, en Europa, a Bélgica, Francia, Suiza y los países Escandinavos. Y en América a: Argentina, Uruguay, Chile, Estados Unidos de América, Panamá, Costa Rica, Brasil, Ecuador y El Salvador. Todos ellos han contribuido a la celebración de Congresos y han creado instituciones protectoras de la madre y del niño.

Para consolidar los derechos de este último, fijando también su situación relativa en el mundo, la profesora Suiza Englantyne Jebb redactó por primera vez una "Declaración de los Derechos del Niño", que fue proclamada el 24 de diciembre de 1924, retomada por Save The Children (2003):

1. El niño debe ser puesto en aptitud de realizar normalmente su desarrollo físico y espiritual.

2. El niño hambriento debe ser alimentado; el enfermo, asistido; el retrasado en su educación, alentado a proseguirla; el desviado de la buena senda, devuelto a ella; el huérfano y el abandonado deben ser recogidos.

3. El niño debe ser el primero en recibir socorro en toda calamidad pública.

4. El niño debe ser puesto en condiciones de ganarse la vida y ser protegido contra toda clase de explotación.

5. El niño debe ser educado inculcándole el sentimiento del deber que él tiene, de utilizar sus mejores cualidades para el servicio de sus hermanos (p. 2).

Además de esta declaración, que surgió del seno de la Sociedad de las Naciones, ha habido otras, que se han producido al celebrarse reuniones tendientes al estudio o a la búsqueda de solución para los problemas de la infancia. 
Rodríguez (citado por Save The Children, 2003), del Instituto Americano de Protección a la Infancia de Montevideo, Uruguay, es autor del siguiente decálogo referente a los Derechos del Niño:

1. "Derecho a la vida.

2. Derecho a la educación.

3. Derecho a la educación especializada

4. Derecho a mantener y desarrollar la propia personalidad.

5. Derecho a la nutrición completa.

6. Derecho a la Tierra

7. Derecho a la Consideración Social.

8. Derecho a la alegría.

9. Derecho a la asistencia económica completa.

10. La suma de todos los anteriores derechos, que viene a formar un derecho integral; el derecho a la vida" (p. 7).

Cada uno de estos derechos, proclamados en una forma general, tiene una serie de accesorios que vienen a darles un sentido completo, sin dejar resquicio a malas o incompletas interpretaciones.

Hay otras declaraciones que merecen ser citadas: El Decálogo de los Derechos del Niño, formulado por el Cuerpo Médico de Chile en mayo de 1928: el Código Panamericano adoptado en 1924 en el Primer Congreso Internacional de Economía Social, llevado a cabo en Buenos Aires, Argentina; y el del Congreso Científico Panamericano de 1925, celebrado en Perú, del que es autor el Doctor Guillermo Sherwell.

También, en el año 1930, en Costa Rica, el Profesor don Luis Felipe González Flores, hizo la Declaración de los Derechos del Niño Costarricense, citada en el acápite siguiente.

Algunos aspectos han ocupado un lugar preponderante entre las preocupaciones de profesionales de distintas áreas y los legisladores en lo que a problemas de la niñez y adolescencia se refiere: el abandono de personas menores de edad y su educación, la delincuencia y el trabajo infantil: la adopción, entre otras.

Los problemas de la minoridad, analizados someramente en líneas precedentes y de los cuales se dijo que eran generales en el mundo, han afectado a Costa Rica en múltiples aspectos.

Nuestro país, de manera plausible, se preocupó por la enseñanza desde fines del siglo pasado, lo que destacó mundialmente en asuntos de enseñanza. No obstante, fuera de legislar en pro de la niñez desde el punto de vista educativo, ninguna otra norma enmienda situaciones peligrosas para la degeneración de nuestra ciudadanía, tales como la mortalidad infantil, que llegó a alcanzar la cifra de 314 por cada mil nacimientos, o sea, el 31,4\%. También hubo épocas en que las estadísticas de nuestra patria arrojaron el 50\% de mortalidad infantil, dentro de la mortalidad general. Desde el punto de vista médico, la situación del niño y la niña costarriwcenses era igualmente lamentable. Azotes como la tuberculosis, la sífilis, la malaria y el alcoholismo, hacían sus estragos en nuestros niños y niñas sin que las arcaicas legislaciones del país se hubieren tomado la preocupación de combatirlas.

En el campo jurídico, nuestra legislación, inspirada en el Código Napoleónico, reconocía en forma draconiana los derechos del padre y la madre, e ignoraba por completo los de los niños, niñas y adolescentes. Acogía sin reservas conceptos tan deplorables como el que se le atribuye a Napoleón Bonaparte "...a la sociedad no le interesa que los bastardos sean reconocidos..." (Prada, 1980, p. 7). 
Los niños, niñas y adolescentes costarricenses estaban expuestos, si delinquían, a ser juzgados con la misma severidad que un adulto; a purgar su error en cárceles públicas y en convivencia con hombres y mujeres curtidos por la delincuencia.

No había entonces, como ahora, una legislación apropiada para el trabajo de personas menores de edad. Las niñas y niños estaban expuestos a ser víctimas de improvisados tutores, cuando la suerte los privaba de sus padres y tenían alguna herencia. Muchos de estos bienes de personas menores de edad fueron a parar a manos inescrupulosas, sin que nadie se preocupara después de su desamparo.

En el caso de personas menores de edad abandonadas, ninguna ley ni organismo, intervenía para impedir que las niñas, niños y adolescentes fueran a parar a hogares en los que, en lugar de encontrar cariño y consideración, eran explotados inhumanamente, castigados con exceso o simplemente humillados por su condición.

Personas con clara visión, enteradas de los progresos sociales en países de tradiciones más antiguas, comprendieron la trascendencia del problema y el peligro de no buscarle solución.

Se comenzó a incubar en nuestro suelo la necesidad de contar con Servicio Social, de dar protección a la niñez, de dar amparo a la madre. En el año 1931, se celebró, bajo su patrimonio, el Primer Congreso Nacional del Niño. Una publicación de aquella época recoge los acontecimientos de mayor trascendencia del evento. Por esas páginas podemos apreciar, las inquietudes que sintieron aquellos hombres por las cuestiones infantiles:

a) Ponencias, análisis sesudos de los diversos problemas, votos, arbitrios y recomendaciones;

b) Sugerencias distintas encaminadas al logro de un feliz derecho positivo que sirviera de norma y de escudo para la niñez; c) Esbozos de tribunales de personas menores de edad, reflexiones orientadas a la represión de la delincuencia, cuando sus agentes eran personas menores de edad; d) Tratamiento específico de los transgresores de la legislación represiva, en el caso de que pudieran ser atribuidas a quienes no hubiesen alcanzado la mayoridad; e) Intervención directa del Patronato Nacional de la Infancia, en los proceso correspondientes; f) Modo de sustituir las penas, según el sentido de que estas concibe el derecho penal, por humanitarias y más modernas medidas de seguridad; g) Establecimiento de la libertad condicional; h) Inquietud por la fundación de casas cuyo principal móvil fuera el de corregir a las personas menores de edad de uno y otro sexos; i) Anhelo de dotar a los niños y niñas sin padre o madre o ambos por la vía de la adopción de una persona que ejerciera cerca de ellos y en su beneficio, los deberes de la paternidad; j) Recomendaciones para que se legislara sobre el trabajo de la madre y el niño ó niña; k) Angustias por la suerte de los hijos e hijas matrimoniales cuando la unión se disolvía por el divorcio; I) Régimen jurídico para los hijos e hijas habidos fuera del lazo nupcial; aspiraciones encaminadas a encauzar la investigación de la paternidad y el aporte de las pruebas a ella atinentes; m) La asistencia social y sus proyecciones en el curso de la vida de los infantes, posibilidades de un seguro maternal; n) Probabilidad de establecer el hogar del niño, con el fin de que allí se recogieran las personas menores de edad sin familia; o) Problemas educativos, con especial atención de la infancia como eje medular alrededor del cual giran las preocupaciones de todos los pedagogos, señal de verdadera distinción de aquel congreso, cuyo seno se vio agitado por los mismos temas que todavía atraen, después de setenta y cinco años de advertidos; p) La fatiga y sus consecuencia en el alma infantil; q) Fomento de los servicios sociales, a efecto de proporcionar nutrición 
completa al niño ó niña; normas para el cálculo de la inteligencia de los escolares, con el propósito de resolver el problema de la baja promoción; r) Sugerencias para que la secretaría de estado, aprovechando los despachos de gobernación y policía, creara un organismo con fuerza legal suficiente para ejercer una censura teatral científicamente organizada y tendiente a vigilar "las exhibiciones que se den para los niños y niñas"; s) Conclusiones de medicina e higiene, que propendían a garantizar la buena salud de la madre y la ventura del fruto de sus entrañas; t) Establecimiento de casas de maternidad, clínicas infantiles, gotas de leche, cocina escolares, colonias veraniegas (Patronato Nacional de la Infancia, 1931, p. 10).

La gloria de aquel evento no está, sin embargo, aislada en el conjunto de méritos ya conquistados por nuestro país. En agosto del año 1956, Costa Rica se vio enaltecida con un acontecimiento de proyecciones internaciones. Concurrieron a este, delegados de las naciones centroamericanas y de Panamá, y su signo peculiar fue una actividad permanente en pro de los problemas que, por modo diverso, afectan el discurrir de la infancia. Lo anterior se dio con la concurrencia de diferentes sectores de profesionales, con ocasión de la celebración de las Bodas de Plata del Patronato Nacional de la Infancia, y cuyo nombre será recogido con beneplácito por la historia de Costa Rica: Primer Congreso Centroamericano-Panameño del Niño.

La Declaración Universal de 1959, hecha por las naciones libres; más que Derechos del Niño puede llamarse Derechos Humanos. Es claro el espíritu democrático que la alienta, y el deseo de hacer conciencia para preservar el género humano del peligro que implican regímenes totalitarios que, irrespetando personalidades, convierten en rebaños a naciones enteras. Ésta contrasta con la Declaración aprobada por el Patronato Nacional de la Infancia el 9 de septiembre de 1930. El régimen de libertad imperante en nuestro sistema, hace innecesario pensar en posibles esclavitudes de cualquiera de las manifestaciones de la personalidad de cada ciudadano. Se considera en ella el problema netamente humano, haciendo caso omiso del aspecto político, y es tan clara que huelga cualquier comentario que sobre ella pretenda hacerse.

\section{CREACIÓN DEL PANI}

El profesor don Luis Felipe González Flores, en mayo de 1929, presentó al Congreso Constitucional un proyecto de ley acompañado de una carta, donde exponía extensamente las razones por las cuales consideraba necesario que en Costa Rica se creara una institución que tuviera por objeto la protección del niño.

El proyecto solicitaba, para la nueva institución, la autonomía y una subvención de cuatro mil colones mensuales. Estas condiciones hicieron que el entonces presidente de la república, don Cleto González Víquez, vetara la ley. Sin embargo, no rechazó la idea totalmente; puso como requisito para aprobarla, el que la institución perteneciera al Estado, y que su subvención alcanzara apenas mil colones mensuales.

El profesor González Flores, antes de ver morir su ideal, se sometió a las condiciones impuestas y el proyecto se convirtió en Ley de la República el quince de agosto de 1930, en virtud de la cual se creó el Patronato Nacional de la Infancia, como una entidad del Estado destinada a velar por el bienestar de la persona menor de edad en su desarrollo físico, intelectual, moral y emocional. 
Después de sancionar la ley, el Presidente González Víquez ofreció todo el apoyo y respaldo del Poder Ejecutivo. El proyecto incluía la obligación de que, pasados dos años, la nueva institución debía presentar un proyecto de Código de la Infancia (Procuraduría General de la República, 1932).

Efectivamente, este fue presentado, y se crea con él disposiciones jurídicas referentes a la Patria Potestad, la Adopción, la Tutela, el Abandono infantil, la obligación de ayuda alimentaria para las personas menores de edad y la administración de bienes de personas menores de edad. Los Derechos del Niño, proclamados por don Luis Felipe, cobran sentido jurídico al emitirse el Código de la Infancia (Procuraduría General de la República, 1932).

Estos tres hechos: la creación del Patronato Nacional de la Infancia, la Declaración de los Derechos del Niño y la instauración del Código de Infancia, sitúan a Costa Rica como uno de los países pioneros, en Latinoamérica y en el mundo, respecto a la protección y atención que se brinda al niño y a la madre.

Una de las primeras preocupaciones del Patronato Nacional de la Infancia fue la vindicación de las hijas e hijos naturales, aquellos quines el Derecho Romano llamó: "Filius Nullis" y el Derecho Consuetudinario Inglés: "Nobody Child" (hijo de nadie).En consecuencia, las personas menores de edad eran víctimas de la discriminación social y juzgados ante la ley en igualdad de condiciones que un adulto.

Recién creado el Patronato Nacional de la Infancia, se vio la necesidad de crear una Junta Directiva. El Presidente de la República preguntó al profesor Luis Felipe González Flores quiénes eran las personas que consideraba más aptas para desempeñar ese cargo. Don Luis Felipe no sólo propuso distinguidos profesionales de diferentes áreas, sino que ofreció desinteresadamente sus servicios, quedando compuesta la directiva por los profesores Don Manuel Obregón, Don Justo Facio, Don Luis Felipe González, el Lic. Alejandro Alvarado Quirós y el Dr. Mario Luján, como miembros propietarios, como suplentes: Don Horacio Acosta, Señorita María Isabel Carvajal (Carmen Lyra) y don Alejandro Montero.

El señor González Flores presentó ante la Directiva un proyecto de Declaración de los Derechos del Niño, que fue aprobado en la sesión del nueve de setiembre de 1930, y la cual reza:

1. "El niño tiene derecho a un patrimonio hereditario, libre de toda clase de taras que le proporcione el máximo de vitalidad favorable a su desenvolvimiento físico y mental.

2. El niño tiene derecho a una gestación normal, garantizada por todos lo medios de que la ciencia dispone, y a que su madre sea protegida para cumplir de la mejor manera posible su función maternal.

3. El niño tiene derecho a sus padres, por lo tanto, a nacer con honor y, cualesquiera que fueran las condiciones de su nacimiento, estar capacitado para el pleno y valioso desarrollo de su vida y de sus actividades sociales.

4. El niño tiene derecho al pecho de su madre y a una nutrición completa hasta el momento en que se le haya capacitado mediante una educación adecuada para que, por sí mismo, pueda ganarse la vida.

5. El niño tiene derecho a la satisfacción de sus necesidades primordiales: alimentación, vestido, curación y todos aquellos métodos higiénicos que aseguran, la conservación y desarrollo de su vitalidad.

6. El niño tiene derecho a no hallarse nunca abandonado moral ni materialmente, a tener un hogar o, en su defecto, a vivir en otro donde sea tratado como hijo. 
7. El niño tiene derecho a vivir sin ser explotado ni en su persona ni en su trabajo, y a que la sociedad lo proteja, dándole facilidad para desarrollar su vida sin que esta esté desnaturalizada.

8. El niño tiene derecho al respeto de todos en la formación de su personalidad, libre de todas aquellas influencias que en alguna forma atenten contra su poder, o que maltraten su conciencia de niño o que perjudiquen su cuerpo.

9. El niño tiene derecho a la máxima educación posible de acuerdo con sus capacidades; a convertirse en un factor de su educación, a desarrollarse siguiendo los intereses propios de su edad y medios educativos adaptados a él.

10. El niño tiene derecho a vivir su infancia, disfrutando de la alegría propia de este período de vida, tiene derecho también a la paz en su hogar y fuera de él, y a que la sociedad le proporcione todas las condiciones necesarias para conservar esa alegría.

11. El niño tiene derecho a ser considerado como irresponsable de sus actos; no hay en consecuencia personas menores de edad delincuentes, sino niños inadaptados, víctimas de la falta de previsión social o niños enfermos que tienen derechos, a más de todo lo establecido para el niño normal, a un tratamiento educativo correccional que los convierta en elementos útiles para la vida colectiva.

12. Todos estos principios integran el derecho del niño a la vida mejorada de la especie y constituyen la base de un anhelo de superación humana y de engrandecimiento nacional. Hacer vivir en la conciencia del país y en la vida diaria estos derechos, constituye la función del Patronato Nacional de la Infancia” (Junta Directiva Patronato Nacional de la Infancia, 1932, p. 6).

Es función del Patronato Nacional de la Infancia concientizar para que estas disposiciones se cumplan en la realidad diaria y no solo en el discurso de conferencias internacionales. Todos estos principios integran el derecho del niño a una vida más justa, y constituyen la base de un anhelo de superación humana y de progreso nacional.

En su inicio, el PANI contaba únicamente con el Departamento Médico y Jurídico. Luego se creó Departamento de Servicio Social, que poco a poco, se amplía.

En la actualidad el PANI, trabaja interdisciplinariamente con profesionales en Derecho, Trabajo Social y Psicología, en atención casuística y en prevención, aparte de contar con amplia normativa legal, como: la Constitución Política, Convención Internacional sobre los Derechos del Niño, el Código de la Niñez y la Adolescencia, el Código de Familia, la Ley Orgánica del PANI, y otras muchas leyes y reglamentos; cuenta también con copiosa jurisprudencia de la Sala Constitucional en materia de Personas Menores de Edad, así como cantidad de Tratados Internacionales en materia de Derechos Humanos.

El Patronato Nacional de la Infancia nació como respuesta visionaria a la impostergable regulación, a cargo del Estado, de las acciones en defensa y protección de madres y niños desvalidos. Fue en los años 30 cuando florecieron las instituciones jurídicas que, en 1999, llegarían a cobrar rango Constitucional.

Una mirada retrospectiva corroborará lo anterior: se inicia la regulación del trabajo de la mujer en estado de gravidez, preferentemente en la Administración Pública, con tímidas incursiones en la empresa privada. Se reguló el monto salarial que la mujer embarazada percibirá durante la incapacidad y el derecho de lactancia. Surgió el Seguro Social de Familia, con un alto 
contenido garante de la alimentación y abrigo de los niños, cuando las circunstancias calificadas imposibilitaren el trabajo de los padres. Se inició la acción del Estado que favorecía la importación y la producción nacional de alimentos y artículos necesarios para nutrirles y abrigarles. Se estimuló la curación de los niños enfermos o que padecieran de "retardo mental". Se calificó la condición de personas menores edad abandonados, vagabundos, mendigos y libertinos. Se instituyó la protección y defensa moral de las personas menores de edad, su salud y su trabajo. Se sentaron las bases que, con ligeros cambios aun hoy se encuentran vigentes, en materia de tratamiento y reubicación de personas menores de edad, reglamentado su depósito provisional y la obligada audiencia al Patronato Nacional de la Infancia en toda acción judicial en que tenga interés una persona menor de edad, so pena de declarar la nulidad de todo lo actuado por solicitud de la Institución.

En 1949, con la promulgación de la Constitución Política de noviembre de ese año, en el Título V de los Derechos y Garantías Sociales, le confió a la Institución “...la protección especial de la madre y el menor... con la colaboración de las otras instituciones del Estado" (Constitución Política de la República 1949).

El 28 de mayo de 1964 se aprobó la Ley Orgánica del Patronato Nacional de la Infancia (Ley $\mathrm{N}^{\circ}$ 3286), reemplazando la Ley $\mathrm{N}^{\circ} 39$ de 15 de agosto de 1930, que fue la de su creación, entró en vigencia, al momento de su publicación en la Gaceta $N^{\circ}$ 139, el 20 de junio de 1964 (Patronato Nacional de la Infancia, 1980, p. 2). En virtud de esta ley, y para poner en práctica las garantías sociales que constituyen su fuente jurídica, se describieron las finalidades de la Institución en siete incisos del artículo quinto, y en diecinueve incisos del artículo sexto, define atribuciones jurídicas para cumplir su cometido constitucional.

La ley fue reglamentada únicamente en lo que se refiere a Bienes de Menores, Salidas del País y el Reglamento de Abandono y Depósito de Menores; este último casi sin aplicación, por estar ren proceso el trámite judicial para las declaratorias de abandono, Reglamento de Reubicación de Menores y Reglamento de Juntas Provinciales. Luego se dicta una nueva Ley Orgánica en 1996 la cual ha sido poco reglamentada.

\section{DEFINICIÓN DEL PATRONATO NACIONAL DE LA INFANCIA}

El Patronato Nacional de la Infancia (PANI) es una persona jurídica, una institución autónoma de carácter público que presta un servicio a los administrados para el bien común; es susceptible de contraer obligaciones y adquirir derechos. Cuenta con una nomenclatura abstracta; diferenciadora de la otra especie de sujetos de derecho que son los individuos humanos, ya que no posee una corporeidad, e incluso, doctrinariamente, se ha considerado persona ficticia.

Dicho ente está conformado por las personas físicas que, con la investidura de servidores públicos, ha trabajado por 65 años con las familias, niños y niñas que más demandan de la sociedad, por sus carencias.

En los últimos 65 años, los funcionarios del Patronato Nacional de la Infancia han prestado a la sociedad costarricense un servicio de valor inconmensurable. Entre los usuarios, existen muchos que valoran el quehacer del servidor y lo gratifican con exposiciones como la siguiente "...para este gran ejército con aprovisionamiento de talentos, verdad y justicia, con la única consigna justicia y derechos para los más débiles y abandonados, los niños. Para este gran ejército de espíritu de bondad, servicio y solidaridad, que defiende los mas elevados valores del ser humano, esencia de su propia naturaleza..." (Patronato Nacional de la Infancia, 1990, p. 1). 
Su labor debe hacernos sentir orgullosos; porque en gran medida, el PANI ha contribuido a impulsar el desarrollo de la niñez y la familia de nuestro país. Por ello, debe vigorizarse su servicio, pues solo la labor responsable y eficaz de cada uno de nosotros deparará a nuestras hijas e hijos un presente y un futuro promisorio.

Los asuntos de la niñez y la adolescencia deben ser motivadores de esa necesaria ayuda y de la relación y coordinación de diferentes instituciones públicas y privadas. Se dará el impulso a proyectos que redunden en beneficio de los niñas, niños y adolescentes, lo que repercutirá (a mediano y largo plazo) en un beneficio cuantificable para la familia, la comunidad y el país entero.

Con las políticas de desarrollo sostenible en armonía con la naturaleza, tomando en cuenta lo económico y lo ecológico, es necesario salvar la naturaleza y protegerla. Así debe ser; pero, la preocupación de salvar a los usufructuarios de esa naturaleza, que están pasando penalidades horrorosas en el nivel mundial: los niños, las niñas y adolescentes.

Es necesario, contar con el compromiso gubernamental en apoyo de cualquier iniciativa en este particular. Los temas de la niñez, la adolescencia y la familia, como elemento fundamental de la sociedad, deben ser motivadores de ese necesario apoyo (...) "Un mundo sin niños sería estéril, sin ilusión. Un mundo de niños tristes y hambrientos es la realidad que estamos viviendo y debemos destruir (...)" (Patronato Nacional de la Infancia, 1931, p. 13).

Si hoy se hace un esfuerzo serio por atender a la niñez, mañana contaremos con adultos preparados para enfrentar el reto en las difíciles condiciones sociales que plantea nuestro tiempo (...) "Donde quiera que las personas de cultura superior dirigen los destinos políticos de una nación y concentren sus miradas en la Infancia, surge radiante la luz que protege al niño desvalido y a la madre desamparada (...)" (Patronato Nacional de la Infancia, 1990, p. 9).

\section{ALGUNAS CARACTERÍSTICAS DEL PAÍS EN LAS DÉCADAS EN QUE LA INSTITUCIÓN HA REALIZADO SU LABOR}

La población costarricense pasó de medio millón de habitantes a cerca de los cuatro millones. La acción estatal ha sido creciente en la actividad social, económica, cultural sanitaria y nutricional. La actividad agrícola aumentó, aun cediendo su importancia a un proceso de industrialización creciente. Se acentúa la dependencia de la economía del exterior en los campos financiero, comercial y tecnológico. Con la irrupción de la televisión a los medios de comunicación colectiva, aumentó la dependencia cultural y la dispersión de valores tradicionales sustituidos por valores de otras sociedades por efecto de demostración. Al amparo del crecimiento del sector público y de la diversificación de la economía nacional hacia lo industrial, emerge una nueva clase media, amplia e influyente. El desarrollo se concentró en el Valle Central. Las principales zonas de pobreza subsisten, principalmente en los cantones rurales, donde los más pobres son Upala, las Cloacas en Alajuela, Barranca, Limoncito y San Felipe de Alajuela, comunidades asumidas por diferentes programas de los gobiernos de turno. Se han producido importantes migraciones campesinas hacia las ciudades, creando focos de miseria y marginalidad social. Se generaron hábitos de consumo que superan la capacidad económica del país.

Es mucho el avance institucional; pero es preciso ajustar el campo de acción a una realidad social, económica y cultural, que ya no es la de hace 65 años, que fue la que vio gestarse y nacer al Patronato Nacional de la Infancia. El PANI debe ajustar su órbita de competencia conforme a las tendencias que muestra la nueva realidad. El papel de la Institución tendió a rectificar el 
desequilibrio de un medio social en el que la economía nacional mostraba una estructura social, predominantemente masculina, que aún en el núcleo familiar constituido, es figura hegemónica. Era pues, natural la creación y desenvolvimiento de una institución que procurara la defensa de las madres y las niñas y niños desvalidos.

No obstante, la realidad social del país es otra. Las instituciones públicas deben efectuar los ajustes necesarios para brindar las respuestas que la nueva situación exige, una respuesta que debe ser más solidaria, que ayude al individuo a alcanzar su plena potencialidad, pero que no lo sustituya; que le haga partícipe de los beneficios del desarrollo económico, social y cultural, pero que no se los regale.

El mundo actual es otro, de ahí la importancia de ser conscientes de la obligación y responsabilidad que se asume al ratificar la Convención sobre los Derechos del Niño desde los diferentes observatorios sociales a involucrar y exigir al Gobierno, los estándares a los que se comprometió con la población menor de edad, y de lo cual debe rendir cuentas cada cinco años al Comité de los Derechos de Niño en Ginebra, Suiza. Asimismo, las Universidades tienen responsabilidad en la sensibilización y capacitación de las y los profesionales en el tema de derechos de las niñas, niños y adolescentes. Se han de crear estructuras para la debida participación real de personas menores de edad, para seguir creciendo en el eje de democracia y paz social.

\section{REFERENCIAS}

Asamblea Legislativa. (1995). Constitución Política de la República de Costa Rica. Versión Actualizada. San José: Publicaciones Jurídicas.

Asamblea Legislativa. (1949). Constitución Política de la República de Costa Rica. San José: Publicaciones Jurídicas.

Junta Directiva Patronato Nacional de la Infancia. (1932). Acuerdo de aprobación de la Declaración de los Derechos del Niño en vía admisnitrativa. Sin publicar.

Patronato Nacional de la Infancia. (1931). Primer Congreso Nacional del Niño. San José: Imprenta Nacional.

Patronato Nacional de la Infancia. (1980). ¿Qué es el PANI?. San José: Taller de Planchas y Suministros.

Patronato Nacional de la Infancia. (1990). Revista Especial 60 Aniversario. San José: Taller de Planchas y Suministros.

Prada, P. (1980). El Ordenamiento Jurídico respecto a la Niñez y al PANI. Sin publicar.

Procuraduría General de la República. (1932). Colección de Leyes y Decretos. Ley número 27 del 25 de octubre. San José: Oficina de Publicaciones.

Save the Children. (2005). Convención sobre los Derechos del Niño. Lima: Editorial IMACOM. 
Save the Children. (2003). Principales Declaraciones de los Derechos del Niño. Lima: Editorial IMACOM.

Vicente R. (1999). Un Vistazo a los antecedentes del Código de Niñez y Adolescencia. San José: Taller de Planchas y Suministros. 